\title{
FLUORIDE LOADED POLYMERIC NANOPARTICLES FOR DENTAL DELIVERY
}

\author{
Sanko Nguyen $^{\mathrm{a},{ }^{*}}$, Carlos Escudero ${ }^{\mathrm{b}}$, Nadia Sediqi ${ }^{\mathrm{a}}$, Gro Smistad ${ }^{\mathrm{a}}$, Marianne Hiorth $^{\mathrm{a}}$ \\ ${ }^{\text {a) }}$ School of Pharmacy, Faculty of Mathematics and Natural Sciences, University of Oslo, P.O. Box \\ 1068, Blindern, 0316 Oslo, Norway

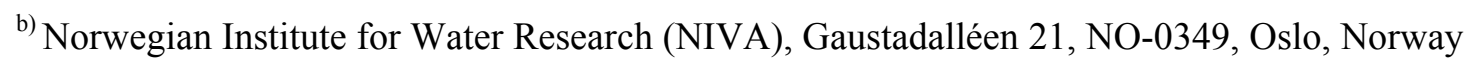 \\ * Corresponding author
}

Sanko Nguyen, Ph.D. P.O. Box 1068, Blindern, 0316 Oslo, Norway Phone: +47 228565 98, Fax: +47 228544 02, E-mail: $\underline{\text { s.h.nguyen@,farmasi.uio.no }}$

European Journal of Pharmaceutical Sciences. 2017.doi:10.1016/j.ejps.2017.04.004@ 2017.

This manuscript version is made available under the CC-BY-NC-ND 4.0 license http://creativecommons.org/licenses/by-nc-nd/4.0/

\begin{abstract}
:
The overall aim of the present paper was to develop fluoride loaded nanoparticles based on the biopolymers chitosan, pectin, and alginate, for use in dental delivery. First, the preparation of nanoparticles in the presence of sodium fluoride $(\mathrm{NaF})$ as the active ingredient by ionic gelation was investigated followed by an evaluation of their drug entrapment and release properties. Chitosan formed stable, spherical, and monodisperse nanoparticles in the presence of $\mathrm{NaF}$ and tripolyphoshate as the crosslinker, whereas alginate and pectin were not able to form any definite nanostructures in similar conditions. The fluoride loading capacity was found to be $33-113 \mathrm{ppm}$, and the entrapment efficiency $3.6-6.2 \%$ for chitosan nanoparticles prepared in $0.2-0.4 \%(\mathrm{w} / \mathrm{w}) \mathrm{NaF}$, respectively. A steady increase in the fluoride release was observed for chitosan nanoparticles prepared in $0.2 \% \mathrm{NaF}$ both in $\mathrm{pH} 5$ and 7 until it reached a maximum at time point 4 hours and maintained at this level for at least
\end{abstract}


24 hours. Similar profiles were observed for formulations prepared in $0.4 \% \mathrm{NaF}$; however the fluoride was released at a higher level at $\mathrm{pH}$ 5. The low concentration, but continuous delivery of fluoride from the chitosan nanoparticles, with possible expedited release in acidic environment, makes these formulations highly promising as dental delivery systems in the protection against caries development.

\section{Introduction}

Fluoride is now recognized to be the most efficient agent to control dental caries mainly through topical effect by inhibiting demineralization and enhancing remineralization of the dental hard tissues (Buzalaf et al., 2011). Despite its well-established caries-protective role, conventional self-care products of fluoride, such as tooth pastes and mouth rinses, is associated with short oral residence time due to the constant cleansing action of saliva. This unfavorable, but physiologically important function of saliva makes it difficult to maintain cariostatic concentrations of fluoride in the oral fluids leading to suboptimal fluoride regimens particularly in patients at elevated risk of developing caries (Fontana and Zero, 2006). A study on the remineralization effect of low-concentration fluoride rinse by Chow et al. concluded that the effectiveness of a fluoride regimen depends less on the dose and more on the ability of the treatment to utilize fluoride efficiently for remineralization (Chow et al., 2002). In addressing the inefficient use of fluoride, improved methods of fluoride delivery to the teeth are sorely needed.

Fluoridated gels and varnishes have been introduced to the commercial market to prolong the contact time between fluoride and tooth surfaces (Pessan et al., 2011b). However, these modalities often involve professional application and contain a much higher concentration of fluoride carrying the risk of excessive fluoride intake. Other fluoride-releasing formats are bioadhesive tablets which have been developed to release fluoride in a sustained manner 
(Bottenberg et al., 1998; Owens et al., 2005). Being applied to the oral mucosa, these dosage forms can cause local irritation and are often poorly tolerated by the patients. Materials for dental restorations have also been incorporated with fluoride in order to protect against recurrent caries following a restoration placement (Wiegand et al., 2007). Despite acting conveniently as fluoride reservoirs, the insertion of these materials requires professional intervention and is limited to patients at risk for restoration failure. More recently, advanced systems, such as microparticles, have been investigated for the controlled delivery of fluoride. In vitro studies showed that biocompatible polymeric microparticles could be used as drug delivery systems to enhance fluoride retention in the oral cavity and promote its timedependent release from potentially different oral care products (de Francisco et al., 2013; Keegan et al., 2012). Apparently, the physical size of the systems plays a key role in oral cavity retention because mouthfeel and the tendency of being sensed are critical factors for patient acceptability and compliance. With this in mind, advancing into the nanoscaled size range would offer even more benefits based on the unique size-dependent properties of particles, including high surface area to volume ratio, and the ability to penetrate biofilm (Allaker, 2010; Wang et al., 2015) and biomimic oral processes (Hannig and Hannig, 2010; Hannig and Hannig, 2012). Constructing nanoparticles with bioadhesive polymers would allow more efficient use of fluoride by increasing its local concentration at the dental hard tissues while evading rapid salivary clearance and excess consumption of the mineral, thus, minimizing systemic effects.

Several methods have been developed to prepare polymeric nanoparticles (Reis et al., 2006). One of the simplest and mildest procedures is based on ionic gelation involving electrostatic complexation between the polymer and an oppositely charged species, often of low molecular weight such as $\mathrm{CaCl}_{2}, \mathrm{ZnCl}_{2}$, and the polyanion tripolyphosphate (TPP) (Grenha, 2012; Jonassen et al., 2013; Paques et al., 2014). Due to the crosslinking effect when the two 
aqueous solutions are mixed, the polymer precipitates and forms nanosized particles. The characteristics of the structures prepared by this method are dependent on several formulation variables, including polymer molecular weight and concentration, and crosslinker to polymer ratio. Recent studies have reported that more compact and smaller nanoparticles with narrower size distributions were produced in the presence of moderate amount of salt due to the salt-induced screening effect (Huang and Lapitsky, 2011; Jonassen et al., 2012; Jonassen et al., 2013; Pistone et al., 2015). This shows in fact that the ionic strength of the solvent is also an important parameter to address in the preparation of biopolymeric nanoparticles by ionic gelation. Giving this background, the most common fluoride compound used in caries prophylaxis is sodium fluoride. In terms of drug properties, this is an unconventional drug molecule to be entrapped in a hydrophilic carrier having very low molecular weight (41.99 $\mathrm{g} / \mathrm{mol})$ and high water solubility $\left(4.3 \mathrm{~g} / 100 \mathrm{ml}\right.$ at $\left.25^{\circ} \mathrm{C}\right)$. Being also a monovalent salt itself, the drug will affect the ionic strength of the solution and thereby the formation of the nanoparticles. With the overall aim of developing fluoride loaded nanoparticles for use in dental delivery, this study will first investigate the preparation of polymeric nanoparticles in the presence of $\mathrm{NaF}$ by ionic gelation followed by an evaluation of their function as fluoride delivery systems in terms of drug entrapment and drug release. Three biopolymers, i.e. chitosan, pectin, and alginate, were chosen for the present investigation due to their recognized bioadhesivity, biocompatibility, biodegradability, and low toxicity (Liu et al., 2008).

\section{Materials \& Methods}

\subsection{Materials}

Amidated pectin (Genu pectin LM-102 AS) was obtained from CPKelco (Lille Skensved, Denmark), and ultrapure chitosan chloride (Protasan ${ }^{\circledR}$ UP CL 213, Novamatrix) and sodium 
alginate (Protanal ${ }^{\circledR}$ LF 10/60) were both obtained from FMC Biopolymer (Norway). Characteristics of the polymers are shown in Table 1. Sodium alginate and amidated pectin (AM-pectin) were purified in-house by dialysis using Spectra/Por 6 membranes (Spectrum Laboratories Inc, CA, USA) with MWCO $8 \mathrm{kDa}$, then freeze-dried and stored in the refrigerator until further use. Tripolyphosphate (TPP, sodium triphosphate pentabasic), $\mathrm{ZnCl}_{2}$, and $\mathrm{NaF}$ were supplied from Sigma-Aldrich (Germany), sodium phosphate monohydrate $\left(\mathrm{NaH}_{2} \mathrm{PO} 4 \times \mathrm{H}_{2} \mathrm{O}\right)$ and disodium phosphate dihydrate $\left(\mathrm{Na}_{2} \mathrm{HPO} 4 \times 2 \mathrm{H}_{2} \mathrm{O}\right)$ from Merck (Darmstadt, Germany), and $\mathrm{NaCl}$ (BDH Prolabo $\left.{ }^{\circledR}\right)$ from VWR Chemicals (Leuven, Belgium). All chemicals were of analytical grade and used as received. All solutions were prepared with Milli-Q purified (Millipore, Molsheim, France) and filtered (0.22 $\mu \mathrm{m}$, Millipak ${ }^{\circledR}$ 40, Millipore) water.

\subsection{Preparation of fluoride loaded nanoparticles}

Fluoride loaded polymeric nanoparticles were prepared by ionic gelation in Milli-Q water by means of a peristaltic pump (Watson-Marlow 520S IP3, Cornwall, UK). First, $15 \mathrm{~g}$ of NaF solution was added dropwise at $25 \mathrm{rpm}$ pumping speed to $45 \mathrm{~g}$ polymeric solution under magnetic stirring, followed by $15 \mathrm{~g}$ of crosslinker solution added in the same manner. Total stirring time for each mixing was 10 minutes. The polymer solutions were filtered $0.8 \mu \mathrm{m}$, and the $\mathrm{NaF}$ and crosslinker solutions were filtered $0.22 \mu \mathrm{m}$ with syringe filters prior to mixing. In the preliminary experiments, the polymer were first dissolved in different solvents (Table 2) before adding the crosslinker solution to the polymer solution in the same manner as previously described. All formulations were allowed to stabilize in room temperature $\left(\sim 20^{\circ} \mathrm{C}\right)$ overnight prior to characterization. 


\subsection{Characterization of nanoparticles}

\section{$\underline{\text { Size and size-related parameters }}$}

The hydrodynamic mean diameter and the polydispersity index (PDI) of the nanoparticles together with the derived count rate were determined by dynamic light scattering (DLS) using a Zetasizer Nano ZS (Malvern Instruments Ltd, Worcestershire, UK). The derived count rate, corresponding to the number of photons detected per second, was used as an expression for the intensity of scattered light (Huang and Lapitsky, 2012; Pistone et al., 2015). All measurements were performed at $25^{\circ} \mathrm{C}$ with backscatter detection and scattering angle of $173^{\circ}$. The refractive index and the viscosity of pure water at $25^{\circ} \mathrm{C}$ were used as calculation parameters. Each sample was measured in triplicate using analysis model for general purpose. Imaging

Atomic force microscopy (AFM) imaging of the nanoparticles was performed using a NanoWizard $\AA$ AFM system (JPK Instruments AG, Germany) with NSC35/AlBS Ultrasharp Silicon Cantilevers (MikroMasch, Spain) through intermittent contact mode in air. The system was set up on an Eclipse TE2000-S inverted optical microscope (Nikon Instruments Inc., Japan), mounted on an anti-vibration table (Halcyonics MOD-1 M plus, Accurion GmbH, Germany) inside a JPK acoustic enclosure. $10 \mu 1$ of the nanoparticle suspension was pipetted onto freshly cleaved mica. After $30 \mathrm{~s}$ adsorption time, excess liquid was removed with a filter paper applied at the edge of the mica. Excess nanoparticles deposited on the mica were removed by rinsing the samples three times with $10 \mu 1$ of Milli-Q water. The samples were allowed to air-dry at room temperature overnight prior to imaging. 


\subsection{In vitro stability}

The fluoride containing nanoparticles were stored as suspensions in the refrigerator and their in vitro stability was studied for one month. At specific time points, the nanoformulations were characterized in terms of particle size and PDI described in the preceding section.

\subsection{Fluoride content}

\section{Drug loading}

$10 \mathrm{ml}$ of the sample suspensions were transferred to pre-washed Spin-X® UF centrifugal concentrators (Corning ${ }^{\circledR}$, NY, USA) with 30k MWCO PES membrane for ultrafiltration and centrifuged for $10 \mathrm{~min}$ at $5000 \mathrm{rcf}\left(20^{\circ} \mathrm{C}\right)$. This step was repeated until the volume of collected filtrate was sufficient for analysis using a fluoride ion selective electrode (ISE, Orion ionplus 9609, Thermo Fisher Scientific, MA, USA) coupled with an ion meter (Orion 720A, MA, USA). In order to provide a constant background ionic strength, $0.5 \mathrm{ml}$ of Total Ionic Strength Adjustment Buffer (TISAB III, EMD Millipore, Darmstadt, Germany) was added to $5 \mathrm{ml}$ of each sample and standard (based on prepared $\mathrm{NaF}$ solutions). Prior to measurements, the electrode operation (slope) was checked according to the manufacturer's instructions. All measurements were performed in plastic beakers under magnetic stirring at $900 \mathrm{rpm}\left(\sim 20^{\circ} \mathrm{C}\right)$. Samples were measured in triplicate and the fluoride concentration (in ppm) was found employing a calibration curve in the appropriate concentration range. The amount of fluoride entrapped in the formulation, i.e. the drug loading (DL), was calculated using the following formula:

$$
F_{\text {entrapped }}(D L)=F_{\text {total }}-F_{\text {free }}
$$

where $F_{\text {total }}$ is the total fluoride content in the formulation converted from accurately recorded weights of $\mathrm{NaF}$ (conversion factor 2.2) and $\mathrm{F}_{\text {free }}$ is the free fluoride content in the sample, i.e. not entrapped in the nanoparticles, determined by ISE measurements. In other words, the drug 
loading expresses the amount of fluoride that is entrapped in each gram nanoparticle suspension (in $\mu \mathrm{g} / \mathrm{g}$ or $\mathrm{ppm}$ ).

\section{Drug entrapment efficiency}

In order to describe the efficiency of the preparation method to incorporate fluoride into the nanoparticles, the fluoride entrapment efficiency (\% EE) was also calculated using the following formula:

$$
\% E E=\frac{\text { Ftotal }- \text { Ffree }}{\text { Ftotal }} \boldsymbol{x} 100
$$

where $\mathrm{F}_{\text {total }}$ and $\mathrm{F}_{\text {free }}$ is the same as described above.

\subsection{In vitro drug release}

In order to separate fluoride loaded nanoparticles from unentrapped (free) fluoride, the sample suspensions were first gelfiltrated using prepacked PD-10 desalting columns (GE Healthcare Bio-Sciences AB, Uppsala, Sweden) containing Sephadex ${ }^{\mathrm{TM}}$ G-25 medium. The columns were equilibrated according to the manufacturer's instructions prior to sample application $(2.5$ $\mathrm{ml})$. The elution medium used was $10 \mathrm{mM}$ phosphate buffer $(3.5 \mathrm{ml})$ either $\mathrm{pH} 5$ or 7 . The particle size and PDI of the nanoparticles were measured before and immediately after gelfiltration to control their intact structure during the process of separation and solvent exchange. $1 \mathrm{ml}$ gelfiltrated samples were then transferred to ready-to-use dialysis devices (Spectra/Por ${ }^{\circledR}$ Float-a-Lyzer G2, Spectrum Laboratories Inc., USA) consisting of cellulose ester membranes with MWCO $20 \mathrm{kDa}$ which were placed in plastic beakers containing 150 $\mathrm{ml}$ of the same medium used for elution. The beakers were covered with parafilm and placed in an orbital shaker-incubator (ES-20, Sia Biosan, Riga, Latvia) set at $100 \mathrm{rpm}$ and $35.0^{\circ} \mathrm{C}$. At certain time intervals, $5 \mathrm{ml}$ aliquots were withdrawn from the beakers and the same amount replaced with fresh medium to maintain sink conditions. Samples were withdrawn in triplicate and analyzed by ion chromatography with suppressed conductivity detection 
(Dionex, Thermo Fisher Scientific). Elution was performed in isocratic mode with a $\mathrm{KOH}$ mobile phase $17 \mathrm{mM}$ and $0.25 \mathrm{~mL} / \mathrm{min}$ flow rate. Samples were diluted 20 times with Milli-Q water and $5 \mu$ injected (loop injection) into the instrument. The separation was performed by a Dionex IonPac AS 18 column and a Guard column Dionex Ion Pac AG18. Chromatographic separation was performed under thermostatic conditions $\left(30^{\circ} \mathrm{C}\right)$ and the temperature of the conductivity detector was set at $35^{\circ} \mathrm{C}$. All the chromatographic data was processed through the software Chromeleon ${ }^{\circledR} 7$ (Thermo Fisher Scientific). Calibration was performed by linear regression with eight $\mathrm{NaF}$ standards in the range 2-1000 $\mu \mathrm{g} \mathrm{F} / \mathrm{L}$. The amount of fluoride released from the nanoparticles is given as the average fluoride concentration detected in the release medium at a particular time point.

\section{Results}

\section{Preparation of fluoride loaded nanoparticles}

Chitosan nanoparticles in different solvents were first prepared and characterized in order to compare the formation of nanoparticles in the presence of $\mathrm{NaF}$. As an early trial, only one type of polymer was tested and the formulation parameters (polymer concentration, polymer to crosslinker ratio, and ionic strength) were selected based on a previous study on the influence of preparation parameters on ionic gelation of chitosan nanoparticles (Jonassen et al., 2012). The results clearly show that only in the presence of the crosslinker TPP and a monovalent salt (either $\mathrm{NaCl}$ or $\mathrm{NaF})$, chitosan formed small nanoparticles $(\sim 100 \mathrm{~nm})$ with low PDIs (0.1 - 0.2) and monomodal size distributions (Table 2). The intensity of scattered light was also significantly higher with these combinations $\left(\sim 10^{4} \mathrm{kcps}\right)$ indicating increased chain associations and particle formation.

In order to study NaF's effect on the formation of different types of polymer based nanoparticles prepared by ionic gelation, alginate and pectin were included in a new set of 
experiments. Switching its view from being a salt to functioning as a drug, the concentration of $\mathrm{NaF}$ was therefore converted from molarity (denoting ionic strength) to percentage $(\mathrm{w} / \mathrm{w})$ to denote the strength of the active substance in the formulation. Without changing the polymer to crosslinker ratio, higher polymer and $\mathrm{NaF}$ concentrations were tested in these experiments with the purpose to increase polymer associations with fluoride, i.e. obtain high drug loading in the formulation, without disturbing the process of particle formation. Thus, six different formulations with regard to polymer type and $\mathrm{NaF}$ concentration were synthesized allowing both inter- and intragroup comparisons. The results showed that pectin and chitosan were able to form nanoparticles with particle sizes in the range of $100-400 \mathrm{~nm}$, low PDIs $(0.1-0.3)$, and monomodal size distributions independent on the NaF concentration tested (Table 3). The situation was, however, completely different for alginate. Although the particle sizes observed were apparently small $(\sim 120 \mathrm{~nm})$, these values are not reliable when interpreted alone. The PDIs for alginate was approximately 1, the size distribution plots revealed multimodality, and the intensities of scattered light were significantly lower (Table 3). Interestingly, the exact same results were obtained for both concentrations of $\mathrm{NaF}$ in the alginate formulations. Thus, the size-related parameters strongly indicate that alginate was unable to form nanoparticles in the presence of the chosen concentrations of the crosslinker and $\mathrm{NaF}$, and so these formulations were therefore excluded from further investigation.

A closer look on the so-formed fluoride loaded pectin and chitosan nanoparticles revealed the formation of two distinct structures. Pectin based nanoparticles were larger $(\sim 400 \mathrm{~nm})$ with higher PDI values (0.3) than the chitosan based nanoparticles ( $100 \mathrm{~nm}$, PDI 0.1-0.2). Moreover, the size distribution plots for pectin based nanoparticles disclosed tailing peaks (Table 3) which prompted further morphology studies using imaging (AFM). Figure 1 clearly displays two contrasting images for the two different polymers. Chitosan features spherical, discrete nanoparticles evenly dispersed throughout the sample (Fig. 1a), whereas pectin 
appears more clustered at some areas. These formations seem more elongated rather than spherical in shape (Fig. 1b). The overall view presents chitosan as compact and monodispersed particulate structures in contrast to pectin which seems to be unorganized, more aggregated and network associated.

\section{In vitro stability}

When stored in the refrigerator as suspensions, practically no changes were observed for fluoride containing chitosan nanoparticles with regard to their particle size (Fig. 2a) and PDI (Fig. 2b). This applied to both concentrations of fluoride in the chitosan formulations. On the contrary, for pectin nanoparticles at both concentrations of fluoride, a slight reduction in the particle size (20-35 nm, Fig. 2a) and the PDI values (Fig. 2b) were observed after a week. This level was then maintained for both parameters for the rest of the tested time period of 39 days.

\section{Fluoride content and release}

Only chitosan successfully formed stable, monodisperse nanoparticles in the presence of a crosslinker and NaF. This polymer was therefore chosen for further investigation for its ability to incorporate and release fluoride. Two concentrations of $\mathrm{NaF}$ in the formulations were studied $(0.2$ and $0.4 \% \mathrm{w} / \mathrm{w})$. The drug loading capacities were found to be 33 and 113 $\operatorname{ppm}(\mu \mathrm{g} / \mathrm{g})$ fluoride for chitosan nanoparticles prepared in $0.2 \% \mathrm{NaF}$ and $0.4 \% \mathrm{NaF}$, respectively. The fluoride entrapment efficiencies (\%EE) were 3.6 and $6.2 \%$ for chitosan nanoparticles prepared in $0.2 \% \mathrm{NaF}$ and $0.4 \% \mathrm{NaF}$, respectively.

In relation to the release study, DLS measurements were used to study the immediate effect of gelfiltration and solvent exchange on the chitosan nanoparticulate structure. Practically no changes in the size and PDI values were detected before and after gelfiltration (data not 
shown). Gelfiltration was therefore considered a mild and appropriate separation method to use because the nanostructures remained well preserved. Changing the solvent from pure water to phosphate buffer $\mathrm{pH} 5$ and $\mathrm{pH} 7$ does not either seem to affect the so-formed nanoparticles.

Traditionally, fluoride has been quantified by means of ISE measurements. In the present study, ion chromatography with suppressed conductivity detector was also employed for fluoride analysis which is an extremely sensitive method having limit of detection as low as $0.1 \mathrm{ppb}$. This was particularly useful in the in vitro release study in order to directly detect very small amounts of fluoride. For chitosan nanoparticles prepared in $0.2 \% \mathrm{NaF}$, the fluoride release profile in both pH 5 and 7 increased steadily until it reached a maximum at time point 4 hours and maintained at this level for 24 hours (Fig. 3a). For chitosan nanoparticles prepared in $0.4 \% \mathrm{NaF}$, similar profiles were observed (Fig. 3b); however, the amount released were higher (almost doubled) and seem to be dependent on the $\mathrm{pH}$ in the release medium. Fig. $3 \mathrm{~b}$ shows that fluoride was released at a higher level in $\mathrm{pH} 5$ compared to $\mathrm{pH} 7$ from formulations prepared in $0.4 \% \mathrm{NaF}$ at almost all time points.

\section{Discussion}

\section{Formation of fluoride loaded nanoparticles}

In the development of fluoride loaded nanoparticles for dental delivery, a biomimicking strategy has been pursued in order to obtain the desired physicochemical properties of the delivery systems. In normal in vivo conditions of the oral cavity, a thin layer called the acquired enamel pellicle is formed on the dental enamel when salivary proteins are adsorbed on the enamel surfaces. This organic layer serves as a protective barrier for the dental hard tissues against acid attack and erosive wear. The pellicle consists mainly of amphiphilic phosphoproteins that have the ability to associate into micelle-like globules which are 
estimated to be 100-500 nm in size (Rolla and Rykke, 1994; Rykke et al., 1995). The approach was therefore to develop nanoparticles to be in this size range for optimal bioadhesion onto the enamel surfaces of the teeth. The chitosan nanoparticles in the present study were measured to be about $100 \mathrm{~nm}$ (Table 3) and, thus, within the desired size range. When it comes to the surface charge of the delivery systems, the complex and dynamic oral environment makes the choice for an optimal charge property not so straightforward. The dental enamel consists of hydroxyapatite (HA) displaying two binding sites that are thought to be involved in the adsorption and desorption processes: the positively charged $\mathrm{Ca}^{2+}$ ions and the negatively charged $\mathrm{PO}_{4}{ }^{3-}$ ions (Yin et al., 2002). Thus, the HA surface can be considered as amphoteric with the ability to interact with both cations and anions dependent on the $\mathrm{pH}$ and ionic strength of the oral medium. Consequently, electrolytes present in saliva can significantly affect the surface properties and hence the interaction processes of HA. In this case, calcium and phosphate are particularly important because their concentrations in saliva are supersaturated with respect to HA (Dawes, 2008). Calcium ions in saliva can reduce the surface potential of the dental enamel to be less negative thereby minimizing electrostatic repulsions and forming calcium bridges that may promote adhesion of negatively charged molecules to HA (Beyer et al., 2010). Because both positively and negatively charged molecules are able to adsorb to the dental enamel depending on the surrounding environment, adsorption experiments in an oral-like setting have to be conducted in order to find the desired surface charge of the delivery systems. Given this background, it was found necessary to investigate both negatively and positively charged polymers as possible candidates as dental delivery systems.

Previous studies have shown that moderate amount of $\mathrm{NaCl}(0.05 \mathrm{M})$ can be used to control the formation and size distribution of ionically crosslinked nanoparticles based on the biopolymers pectin, alginate, and chitosan (Jonassen et al., 2012; Jonassen et al., 2013; 
Pistone et al., 2015). In this study, $\mathrm{NaF}$ has been introduced mainly as an efficient anticaries agent but also as a possible substitute for $\mathrm{NaCl}$ in such systems. The results obtained (Table 2 and 3) show that $\mathrm{NaF}$ can replace $\mathrm{NaCl}$; however, this was dependent on the type of polymer and the concentration of $\mathrm{NaF}$ used in the formulation. An intergroup comparison (Table 3) shows that alginate was the only polymer not able to form any definite nanostructure. The high PDI values together with very low intensity of scattered light and multimodal intensity size distribution strongly suggest that the alginate chains remain in the network conformation, possibly as loose chain aggregates, rather than organizing into compact polymer coils. Huang and Lapitsky demonstrated that high monovalent salt concentrations (ca $0.5 \mathrm{M}$ ) in a crosslinked polymer system can weaken the binding at the polymer and crosslinker junctions to the point that microgel formation can be disrupted (Huang and Lapitsky, 2011). Although the authors demonstrated this with chitosan, the same situation seems to have taken place in the present alginate system. The formation of colloid alginate particles was inhibited possibly because the level of optimal ionic strength in the system has been overly exceeded owing to high amount of NaF inclusion $(0.12-0.24 \mathrm{M})$. Despite the same ionic strength were tested for chitosan and pectin in the present study, these polymers nevertheless were capable in forming some kind of stable nanostructures indicating the ionic strength optimum in crosslinked polymer systems is different depending on the type of polymer. It is worth mentioning that crosslinker contributions to the total ionic strength in the systems were negligible and so minor differences in the amount of crosslinker present between the polymer systems were considered not to be a determinative factor.

Within moderate levels of ionic strength, the salt-induced screening effect will reduce the electrostatic repulsion between the polymer chains, thereby increasing chain aggregation tendency (dense packing of chains) and the formation of polymer coils (particles). In which degree this effect will occur, seems to rely on the type of associations that exist between the 
polymer and the salt in question. The structures created based on chitosan and pectin systems were clearly different. In the case of chitosan, this polymer was able to fully form the classic example of nanoparticulate structure envisioned as small $(\sim 100 \mathrm{~nm})$, spherical, and uniform nanoparticles with narrow size distribution (Table 3 and Fig. 1a). These results support an efficient charge screening effect upon the addition of $\mathrm{NaF}$ to crosslinked chitosan in agreement with previous reports applying $\mathrm{NaCl}$ in similar systems (Huang and Lapitsky, 2011; Jonassen et al., 2012). In addition, fluorine is the most electronegative and reactive of all elements in the periodic table enabling bonds with other atoms of ionic character. It is therefore highly likely that the charge screening effect is even more efficient with fluoride creating a superior environment for TPP to crosslink and pulling the chitosan chains together into a tighter nanoparticle matrix. As a result of these forces exerted by $\mathrm{NaF}$, small and compact nanoparticles were formed as observed. In the case of pectin, the polymer obtained a morphology characterized by larger chain aggregates combined with filamentous chain alignments (interwoven strands) rather than well-defined particulate structures (Fig. 1b). Due to the unattractive combination of pectin's anionic character and high electronegativity of fluorine, repulsive forces dominate. The salt-induced screening effect is less pronounced compared to that of chitosan and this may explain the larger size $(\sim 400 \mathrm{~nm})$ and broader, tailing size distribution retrieved from DLS measurements for pectin systems (Table 3). Moreover, weak hydrogen bonds may be present between fluoride and $\mathrm{OH}-/ \mathrm{CONH}_{2}$-groups of the amidated pectin chains (Reichenbächer et al., 2005) corresponding to the image of a loose, but relatively stable network connecting the chain aggregates as shown in Fig. $1 \mathrm{~b}$. Presence of stabilizing weak hydrogen bonds is also supported by the in vitro stability data of the pectin based systems. A slight decrease in both the particle size and PDI were observed after a week (Fig. 2a and b) suggesting that stabilization through such associations seems to take somewhat longer time. However after which a steady state has been established, the 
pectin based systems were found remarkably stable in vitro (comparable to the chitosan based systems) for at least one month when stored in the refrigerator. It is important to emphasize here that the fluoride content of the nanoparticles was not directly monitored in the present stability study. However, since the nanoparticles could only be formed in such a stringent system with the ionic strength as a controlling parameter, it was expected that any fluoride leakage from the nanoparticles would lead to changes in the nanoparticulate system and, thus, to detectable changes in the particle size and PDI measurements. In spite of this assumption, it is nevertheless an imperative to monitor the fluoride content in the formulation in order to assess the storage stability of the final pharmaceutical product.

\section{Evaluation of chitosan nanoparticles as fluoride delivery systems}

Since intragroup comparison did not unveil significant differences in the physical characteristics of the chitosan nanoparticles (Table 3), both high $(0.4 \%)$ and low $(0.2 \%) \mathrm{NaF}$ concentrations in the formulations were evaluated for their fluoride delivery properties. Generally, the amount of drug fed into a drug delivery system is positively correlated to the drug loading capacity. This principle does indeed apply in the present delivery systems as the fluoride loading capacity was found to increase from $33 \mathrm{ppm}$ to $113 \mathrm{ppm}$ when the initial $\mathrm{NaF}$ concentration increased from $0.2 \%$ to $0.4 \%$ in the formulations. Suitable dosage forms to administer the developed fluoride loaded nanoparticles could be mouthwash or mouth spray as these products are well tolerated by patients, easy to use for self-care, and may also help to wet and lubricate the oral cavity in addition to deliver fluoride. The latter is particularly useful for patients suffering from xerostomia (hyposalivation) who are concurrently at high risk for caries development. Fluoride mouth rinses sold over-the-counter typically contain $230-900$ ppm fluoride (i.e. $0.05-0.2 \% \mathrm{NaF}$ ) for caries prevention (Pessan et al., 2011a). Higher fluoride strength in liquid-like pharmaceutical formulations either require a prescription or professional application, and are only recommended for patients at high risk of developing 
caries (Weyant et al., 2013). Thus, the developed chitosan nanoformulations contain a quite lower content of fluoride than what is commercially common. This was unexpected since we anticipated strong electrostatic interactions between fluoride and chitosan that would lead to high drug loading capacity. One plausible explanation is that the chitosan nanoparticles were prepared in water where the $\mathrm{pH}$ is close to chitosan's $\mathrm{pKa}$ value enabling the presence of both protonated and deprotonated form of chitosan (lower charge density) at the time of fluoride incorporation. Similar to the drug loading capacity, the fluoride entrapment efficiencies found $(3.6-6.2 \%)$ were also rather low. The fluoride entrapment did increase with increasing initial content of fluoride which is in agreement with previous finding on fluoride loaded chitosan microparticles prepared by spray drying (Keegan et al., 2012). Collectively, we could say that the fluoride loading capacity and entrapment efficiency of the chitosan nanoparticles could be optimized by carefully tune in the preparation parameters having the limitation of the nanosystems' ionic strength in mind.

Despite the apparently low fluoride loading in the chitosan nanoparticles, it is generally recognized that the concentration of fluoride required to enhance remineralization is significantly lower than that required for the inhibition of demineralization. A base line salivary fluoride level of $0.1 \mathrm{ppm}$ has been claimed to give adequate protection against caries progression (Featherstone, 2006). Therefore, it was also essential to assess the performance of the chitosan nanoparticles to release bioavailable fluoride to maintain such low intraoral levels and function as an oral fluoride reservoir. For topical fluoride formulations, the retention of therapeutic intraoral concentrations is of paramount importance due to its premature removal by oral functions. In a study by Eakle et al. salivary fluoride levels was found to return to baseline after approximately 2 hours after the application of a single rinse (Eakle et al., 2004). On the other hand, the release of small solutes, such as fluoride, from hydrogels is commonly too fast due to rapid diffusion to proclaim sustained delivery. The 
chitosan nanoparticles exhibited a biphasic fluoride release profile with a steady increasing discharge within the first 4 hours followed by sustained release for at least 24 hours (Fig. 3). Although the levels of fluoride release were considered quite low (because of low initial drug loading), a prolonged fluoride release pattern was obtained. In fact, only $10-15 \%$ of maximum fluoride that could be released from the chitosan nanoparticles was detected at time point 24 hours. This slow release property is more and more emphasized as several studies have demonstrated that even a low, but sustained level of fluoride in the oral cavity can help to prevent or reverse the caries process (Featherstone, 1999, 2006; Lynch et al., 2004; Pessan et al., 2008). In addition, reduced dosing frequency also increases patient compliance. The mechanism for long-term release of small molecules has been suggested by Lawrence et al. (Lawrence et al., 2016). They demonstrated that high crosslink densities in a polymer matrix, i.e. small mesh size, could serve as strong, physical barriers for diffusion of small solutes. This may also be a valid explanation for the extended release of the prepared chitosan-TPP crosslinked nanoparticles in addition to the strong binding strength present between fluoride and chitosan.

The present in vitro release study was designed to best possible simulate a simplified condition of the oral cavity in a "normal" state, represented by $\mathrm{pH} 7$, and in an acid challenged state, represented by $\mathrm{pH}$. The interpretation of the results in terms of a $\mathrm{pH}$ change becomes highly relevant when developing anti-caries delivery systems because the disease is characterized by destruction of dental hard tissues by acids produced by bacteria metabolizing dietary carbohydrates (Selwitz et al., 2007). A desirable enamel protective response to such an acid attack is to increase the level of bioavailable fluoride at the enamel surface in order to accelerate the repairing process and also to make the enamel more resistant to subsequent acidic challenges by the formation of fluorapatite (Buzalaf et al., 2011; Ten Cate and Featherstone, 1991). There was no difference in the fluoride release from chitosan 
nanoparticles prepared in $0.2 \% \mathrm{NaF}$ in $\mathrm{pH} 5$ and $\mathrm{pH} 7$ (Fig. 3a). However, the fluoride release was expedited in $\mathrm{pH} 5$ compared to $\mathrm{pH} 7$ for chitosan nanoparticles prepared in $0.4 \% \mathrm{NaF}$ (Fig. 3b). Increased fluoride release may be due to increased swelling behavior and in turn particle disintegration of the chitosan nanoparticles when the environmental $\mathrm{pH}$ changes from neutral to acidic (López-León et al., 2005). This effect could not be observed with the formulation prepared in $0.2 \% \mathrm{NaF}$ concentration probably because the amounts of fluoride detected were so low so that it could not reveal any $\mathrm{pH}$-dependent differences. The transition to faster release behavior in acidic $\mathrm{pH}$ is a highly favorable attribute of the nanoparticles in order to function as a responsive fluoride delivery system under a potential cariogenic condition.

Regarding the potential application of the nanoparticles, these formulations have good prospects in the clinics as specific agents for patients at increased risk of developing caries. Because dental caries is a complex and multifactorial disease, many risk indicators have been identified, including eating disorders, xerostomia, mental disabilities, previous caries experience, active orthodontic treatment, and presence of existing restorations and exposed root surfaces (Panel, 2001). Ranging from various medical conditions to socioeconomic status, a large patient population is in fact in need of efficient fluoride products for prophylactic therapy. In these individuals, either a high-content or a low, but sustained fluoride product would be highly beneficial to protect against enamel deterioration. By a simple preparation method, we have developed fluoride loaded chitosan nanoparticles that allowed for prolonged delivery of fluoride for potential use in caries prevention. Apart from the pharmaceutical evaluation point of view, upon releasing fluoride its bioavailability is dependent upon various physiological factors, such as salivary composition and secretion rate. The presence of an oral biofilm on the dental surface (dental plaque) and/or the oral mucosa as competing binding substratum (mucoadhesion) may also intervene with the nanoparticle adhesion and fluoride 
action. Despite promising results presented herein, intraoral effects that may have an impact on the nanoparticles' adhesion onto the enamel and, thus, their intraoral retention should be taken into account in the next step of the developmental work.

\section{Conclusions}

The application of nanoparticles to overcome barriers in drug delivery is a well-recognized approach; however, the use of these platforms in dental pharmacotherapy is yet a less explored area. In the present study, polymer based nanoparticulate formulations have been developed in order to provide new and improved means for efficient topical delivery of fluoride. The biopolymers alginate, pectin, and chitosan were investigated with the monovalent salt sodium fluoride as the active fluoride compound. In the presence of $\mathrm{NaF}$ and an appropriate crosslinker, only chitosan could form stable, monodisperse nanoparticles. Alginate was not able to form nanoparticles because the ionic strength optimum was exceeded at the salt concentrations tested, whereas pectin formed large, undefined nanostructures. The fluoride loading and entrapment efficiency of the chitosan nanoparticles were found to be 33$113 \mathrm{ppm}$ and 3.6-6.2\%, respectively, in the tested conditions. These values can be optimized by carefully adjust the preparation parameters during fluoride incorporation. The present in vitro release data support a low concentration, but continuous delivery of fluoride from the chitosan nanoparticles. The fluoride release seems to increase in an acidic environment when simulating a cariogenic attack. These properties are highly advantageous for dental formulations targeting patients at high risk of developing caries. Although promising results, more investigations into enamel adhesion and intraoral influences are needed in order to scrutinize their prospects in the prevention of dental caries.

\section{Conflict of interest}

The authors declare no conflict of interest. 


\section{Acknowledgements}

This work was supported by a grant from The Research Council of Norway (grant number 231324).

\section{References}

Allaker, R., 2010. The use of nanoparticles to control oral biofilm formation. Journal of dental research 89, 1175-1186.

Beyer, M., Reichert, J., Heurich, E., Jandt, K.D., Sigusch, B.W., 2010. Pectin, alginate and gum arabic polymers reduce citric acid erosion effects on human enamel. dental materials 26, 831-839.

Bottenberg, P., Bultmann, C., Gräber, H., 1998. Distribution of fluoride in the oral cavity after application of a bioadhesive fluoride-releasing tablet. J. Dent. Res. 77, 68-72.

Buzalaf, M.A.R., Pessan, J.P., Honório, H.M., Ten Cate, J.M., 2011. Mechanisms of action of fluoride for caries control, in: Buzalaf, M.A.R. (Ed.), Fluoride and the Oral Environment. Karger, Basel, pp. 97-114.

Chow, L., Takagi, S., Frukhtbeyn, S., Sieck, B., Parry, E., Liao, N., Schumacher, G., Markovic, M., 2002. Remineralization effect of a low-concentration fluoride rinse in an intraoral model. Caries research 36, 136-141.

Dawes, C., 2008. Salivary flow patterns and the health of hard and soft oral tissues. The Journal of the American Dental Association 139, 18S-24S.

de Francisco, L.M.B., Cerquetani, J.A., Bruschi, M.L., 2013. Development and characterization of gelatin and ethylcellulose microparticles designed as platforms to delivery fluoride. Drug Dev. Ind. Pharm. 39, 1644-1650.

Eakle, W.S., Featherstone, J.D., Weintraub, J.A., Shain, S.G., Gansky, S.A., 2004. Salivary fluoride levels following application of fluoride varnish or fluoride rinse. Community dentistry and oral epidemiology 32, 462-469.

Featherstone, J.D., 1999. Prevention and reversal of dental caries: role of low level fluoride.

Community dentistry and oral epidemiology 27, 31-40.

Featherstone, J.D., 2006. Delivery challenges for fluoride, chlorhexidine and xylitol. BMC Oral Health 6,1 .

Fontana, M., Zero, D.T., 2006. Assessing patients' caries risk. The Journal of the American Dental Association 137, 1231-1239.

Grenha, A., 2012. Chitosan nanoparticles: a survey of preparation methods. Journal of drug targeting 20, 291-300.

Hannig, M., Hannig, C., 2010. Nanomaterials in preventive dentistry. Nature nanotechnology 5, 565569.

Hannig, M., Hannig, C., 2012. Nanotechnology and its role in caries therapy. Advances in dental research 24, 53-57.

Huang, Y., Lapitsky, Y., 2011. Monovalent salt enhances colloidal stability during the formation of chitosan/tripolyphosphate microgels. Langmuir 27, 10392-10399.

Huang, Y., Lapitsky, Y., 2012. Salt-assisted mechanistic analysis of chitosan/tripolyphosphate microand nanogel formation. Biomacromolecules 13, 3868-3876.

Jonassen, H., Kjøniksen, A.-L., Hiorth, M., 2012. Effects of ionic strength on the size and compactness of chitosan nanoparticles. Colloid and Polymer Science 290, 919-929.

Jonassen, H., Treves, A., Kjøniksen, A.-L., Smistad, G., Hiorth, M., 2013. Preparation of ionically cross-linked pectin nanoparticles in the presence of chlorides of divalent and monovalent cations. Biomacromolecules 14, 3523-3531.

Keegan, G.M., Smart, J.D., Ingram, M.J., Barnes, L.-M., Burnett, G.R., Rees, G.D., 2012. Chitosan microparticles for the controlled delivery of fluoride. J. Dent. 40, 229-240. 
Lawrence, P.G., Patil, P.S., Leipzig, N.D., Lapitsky, Y., 2016. Ionically Cross-Linked Polymer Networks for the Multiple-Month Release of Small Molecules. ACS applied materials \& interfaces 8, 4323-4335.

Liu, Z., Jiao, Y., Wang, Y., Zhou, C., Zhang, Z., 2008. Polysaccharides-based nanoparticles as drug delivery systems. Advanced drug delivery reviews 60, 1650-1662.

López-León, T., Carvalho, E., Seijo, B., Ortega-Vinuesa, J., Bastos-González, D., 2005.

Physicochemical characterization of chitosan nanoparticles: electrokinetic and stability behavior. Journal of Colloid and Interface Science 283, 344-351.

Lynch, R., Navada, R., Walia, R., 2004. Low-levels of fluoride in plaque and saliva and their effects on the demineralisation and remineralisation of enamel; role of fluoride toothpastes. International dental journal 54, 304-309.

Owens, T.S., Dansereau, R., Sakr, A., 2005. Development and evaluation of extended release bioadhesive sodium fluoride tablets. Int. J. Pharm. 288, 109-122.

Panel, N.I.o.H.C.D., 2001. National Institutes of Health Consensus Development Conference statement: diagnosis and management of dental caries throughout life, March 26-28, 2001. The Journal of the American Dental Association 132, 1153-1161.

Paques, J.P., van der Linden, E., van Rijn, C.J., Sagis, L.M., 2014. Preparation methods of alginate nanoparticles. Advances in colloid and interface science 209, 163-171.

Pessan, J.P., Al-Ibrahim, N.S., Buzalaf, M.A.R., Toumba, K.J., 2008. Slow-release fluoride devices: a literature review. Journal of Applied Oral Science 16, 238-244.

Pessan, J.P., Toumba, K.J., Buzalaf, M.A.R., 2011a. Topical use of fluorides for caries control. Pessan, J.P., Toumba, K.J., Buzalaf, M.A.R., 2011b. Topical use of fluorides for caries control, in: Buzalaf, M.A.R. (Ed.), Fluoride and the Oral Environment. Karger Publishers, Basel, pp. 115-132. Pistone, S., Qoragllu, D., Smistad, G., Hiorth, M., 2015. Formulation and preparation of stable crosslinked alginate-zinc nanoparticles in the presence of a monovalent salt. Soft matter 11, 5765-5774.

Reichenbächer, K., Süss, H.I., Hulliger, J., 2005. Fluorine in crystal engineering - "the little atom that could". Chemical Society Reviews 34, 22-30.

Reis, C.P., Neufeld, R.J., Ribeiro, A.J., Veiga, F., 2006. Nanoencapsulation I. Methods for preparation of drug-loaded polymeric nanoparticles. Nanomedicine: Nanotechnology, Biology and Medicine 2, 821.

Rolla, G., Rykke, M., 1994. Evidence for presence of micelle-like protein globules in human saliva.

Colloids and Surfaces B: Biointerfaces 3, 177-182.

Rykke, M., Smistad, G., Rölla, G., Karlsen, J., 1995. Micelle-like structures in human saliva. Colloids and Surfaces B: Biointerfaces 4, 33-44.

Selwitz, R.H., Ismail, A.I., Pitts, N.B., 2007. Dental caries. The Lancet 369, 51-59.

Ten Cate, J., Featherstone, J., 1991. Mechanistic aspects of the interactions between fluoride and dental enamel. Critical Reviews in Oral Biology \& Medicine 2, 283-296.

Wang, L.-S., Gupta, A., Rotello, V.M., 2015. Nanomaterials for the Treatment of Bacterial Biofilms. ACS Infectious Diseases 2, 3-4.

Weyant, R.J., Tracy, S.L., Anselmo, T., Beltrán-Aguilar, E.D., Donly, K.J., Frese, W.A., Hujoel, P.P., Iafolla, T., Kohn, W., Kumar, J., Levy, S.M., Tinanoff, N., Wright, J.T., Zero, D., Aravamudhan, K., Frantsve-Hawley, J., Meyer, D.M., 2013. Topical fluoride for caries prevention. The Journal of the American Dental Association 144, 1279-1291.

Wiegand, A., Buchalla, W., Attin, T., 2007. Review on fluoride-releasing restorative materialsfluoride release and uptake characteristics, antibacterial activity and influence on caries formation. Dent. Mater. 23, 343-362.

Yin, G., Liu, Z., Zhan, J., Ding, F., Yuan, N., 2002. Impacts of the surface charge property on protein adsorption on hydroxyapatite. Chemical Engineering Journal 87, 181-186. 
Table 1. Characteristics of the polymers used.

Type of polymers

Structure

Chemical compound (composition)

Molecular weight

$\mathrm{pKa}$

Alginate

Degree of amidation (DA); Degree of deacetylation (DDA); Degree of esterification (DE).

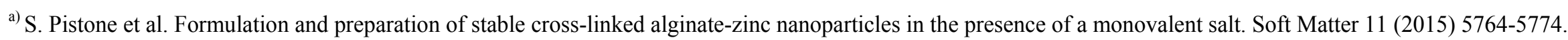

b) H. Jonassen et al. Effects of ionic strength on the size and compactness of chitosan nanoparticles. Colloid Polym Sci 290 (2012) 919-929.

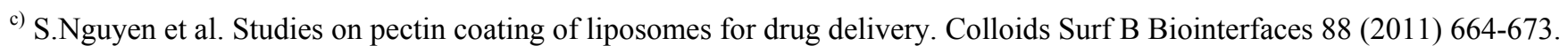


Table 2. Characteristics of chitosan nanoparticles prepared in different solvents (mean \pm standard deviation).

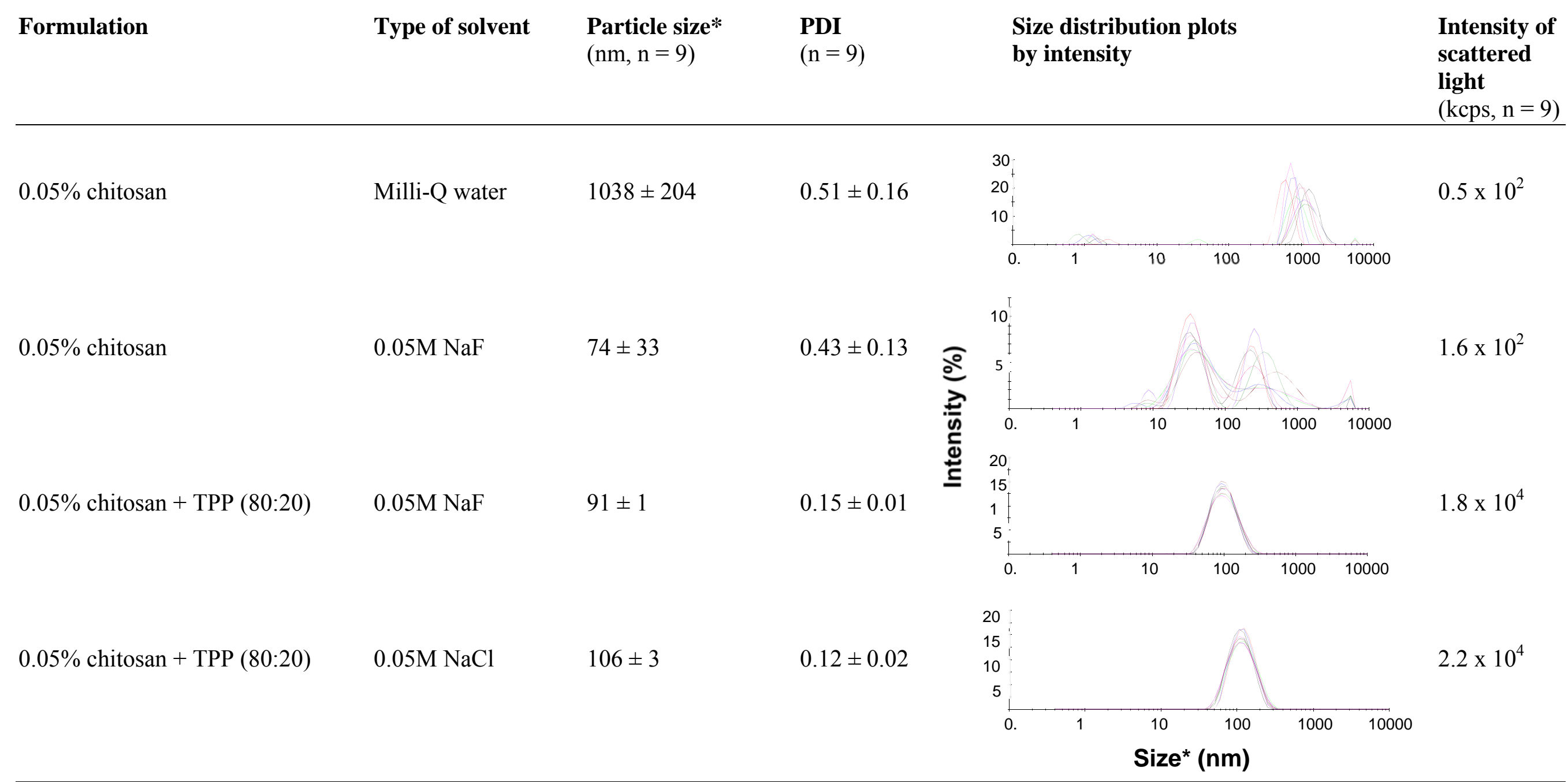

* The particle size was measured as the hydrodynamic mean diameter.

Final concentrations $(\% \mathrm{w} / \mathrm{w})$ of the dispersions are given. Polymer to crosslinker ratios (w/w) are given in parentheses. Based on calculations, $0.05 \mathrm{M} \mathrm{NaF}$ is equivalent to $0.08 \%$ (w/w) NaF. 
Table 3. Characteristics of polymeric nanoparticles prepared in the presence of $\mathrm{NaF}$ (mean \pm standard deviations).

\section{Formulation}

$0.07 \%$ chitosan + TPP $(80: 20)+0.2 \% \mathrm{NaF}$

$0.07 \%$ chitosan $+\mathrm{TPP}(80: 20)+0.4 \% \mathrm{NaF}$

$0.07 \%$ AM-pectin $+\mathrm{ZnCl}_{2}(85: 15)+0.2 \% \mathrm{NaF}$

$0.07 \%$ AM-pectin $+\mathrm{ZnCl}_{2}(85: 15)+0.4 \% \mathrm{NaF}$

$0.07 \%$ alginate $+\mathrm{ZnCl}_{2}(65: 35)+0.2 \% \mathrm{NaF}$

$0.07 \%$ alginate $+\mathrm{ZnCl}_{2}(65: 35)+0.4 \% \mathrm{NaF}$

\section{Particle size*}

(nm)

$\begin{array}{ll}112 \pm 1 & 0.16 \pm 0.02 \\ (\mathrm{n}=9) & (\mathrm{n}=9)\end{array}$

$101 \pm 1$

$(\mathrm{n}=9)$

$407 \pm 8$

$(\mathrm{n}=6)$

$421 \pm 6$

$(\mathrm{n}=6)$

$$
\begin{aligned}
& 117 \pm 12 \\
& (n=6)
\end{aligned}
$$

$0.14 \pm 0.01$

$(\mathrm{n}=9)$

$$
\begin{aligned}
& 0.29 \pm 0.02 \\
& (n=6)
\end{aligned}
$$

$0.30 \pm 0.01$

$(\mathrm{n}=6)$

$0.95 \pm 0.08$

$(\mathrm{n}=6)$

$$
\begin{array}{ll}
117 \pm 13 & 0.95 \pm 0.09 \\
(\mathrm{n}=6) & (\mathrm{n}=6)
\end{array}
$$

Size distribution plots by intensity
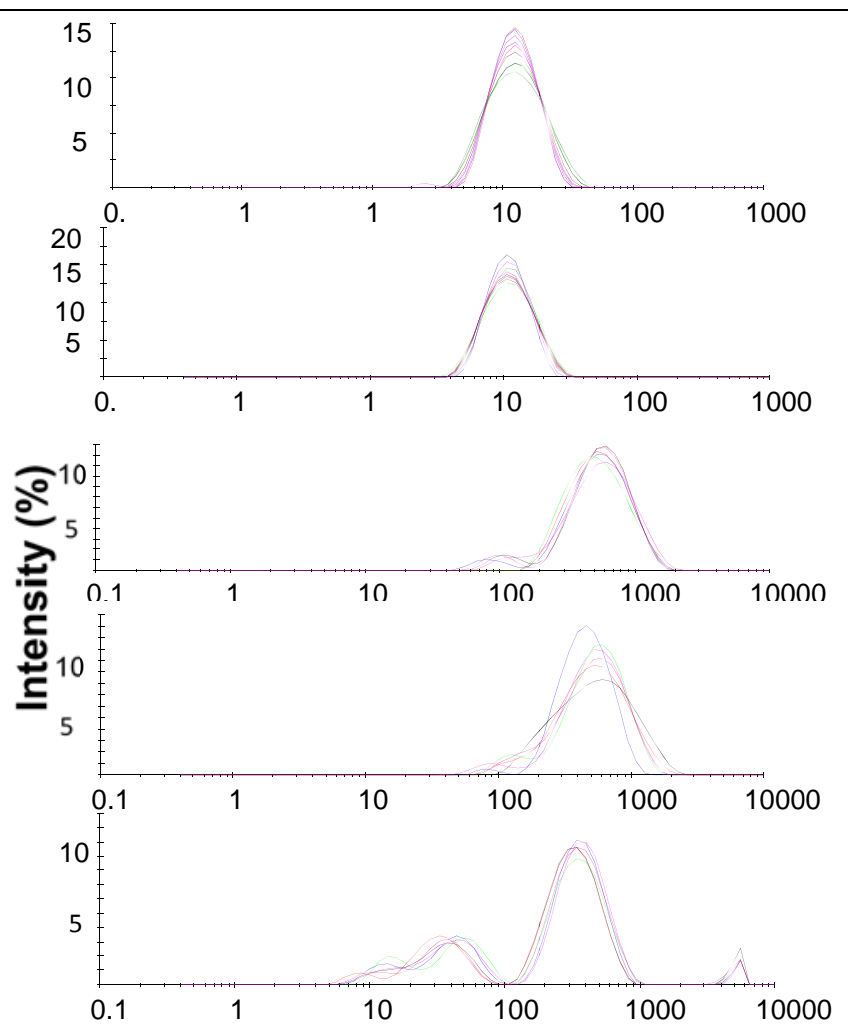

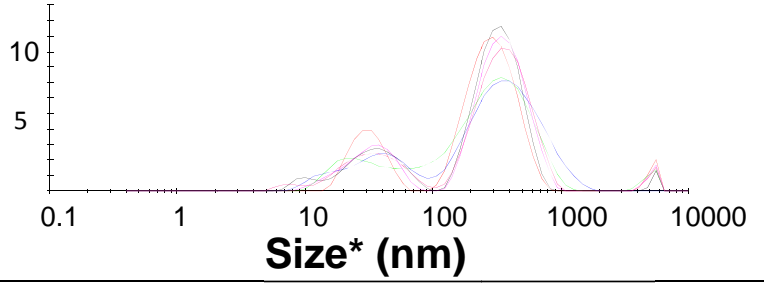

Intensity of scattered light (kcps)

\section{$5.8 \times 10^{4}$}

$(\mathrm{n}=9)$

$2.5 \times 10^{4}$

$(\mathrm{n}=9)$

\section{$1.3 \times 10^{4}$}

$(\mathrm{n}=6)$

$0.9 \times 10^{4}$

$(\mathrm{n}=6)$

$4.1 \times 10^{2}$

$(\mathrm{n}=6)$

$4.5 \times 10^{2}$

$(\mathrm{n}=6)$

* The particle size was measured as the hydrodynamic mean diameter.

Final concentrations $(\% \mathrm{w} / \mathrm{w})$ of the dispersions are given. Polymer to crosslinker ratios $(\mathrm{w} / \mathrm{w})$ are given in parentheses. Based on calculations, the ionic strenght of $0.2 \%$ and $0.4 \%(\mathrm{w} / \mathrm{w}) \mathrm{NaF}$ is equivalent to $0.12 \mathrm{M}$ and $0.24 \mathrm{M}$, respectively. 


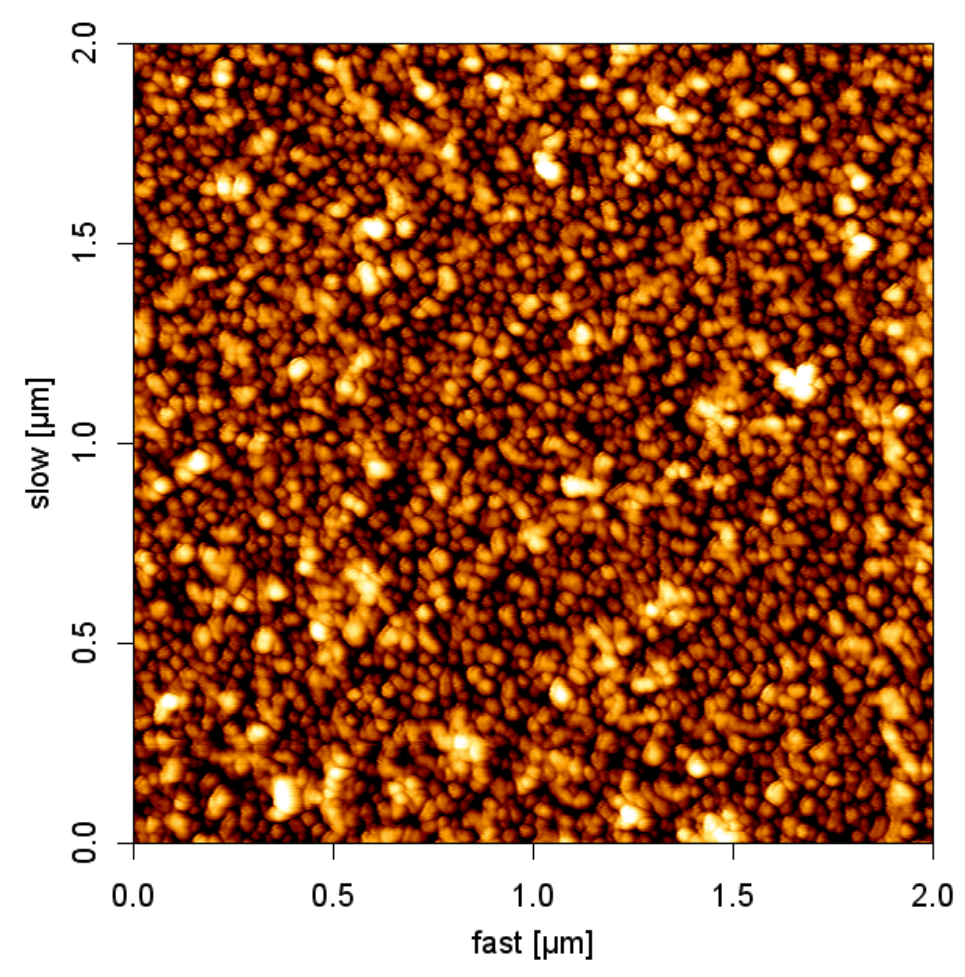

a)

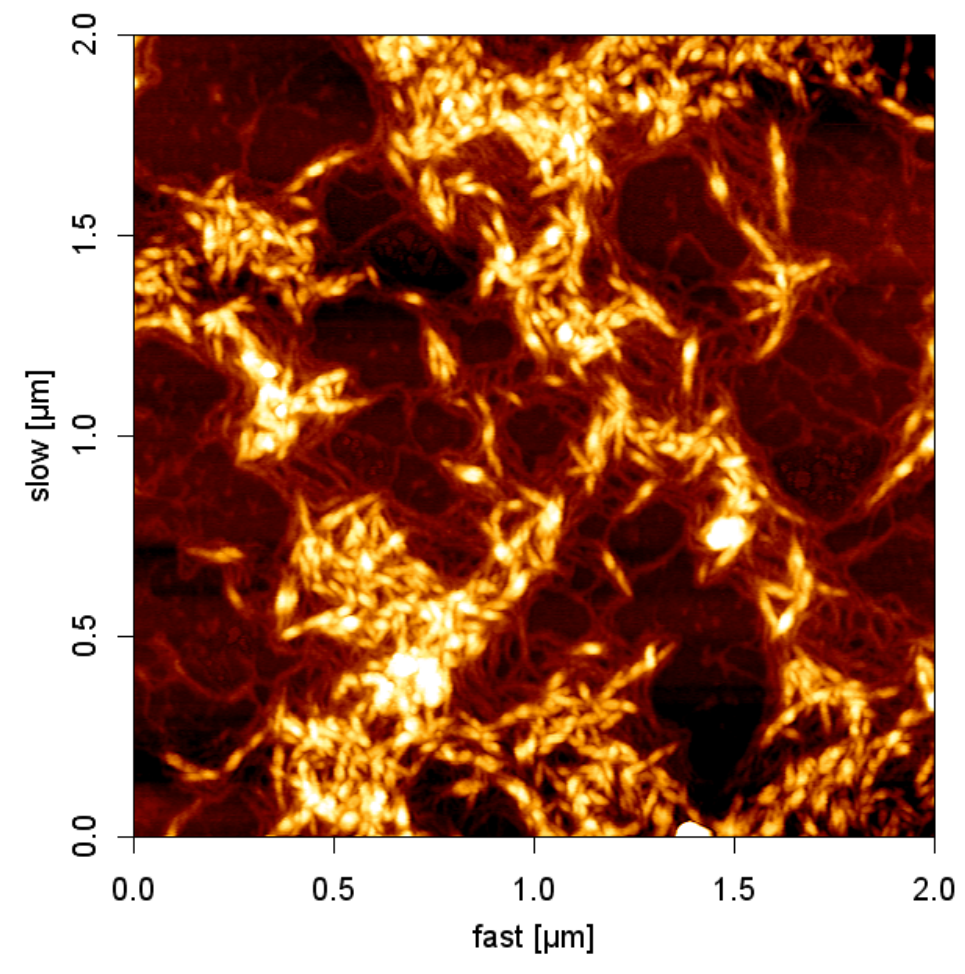

Figure 1: Representative AFM images of a) chitosan and b) AM-pectin nanoparticles prepared in $0.2 \% \mathrm{NaF}$ and the appropriate crosslinker. 


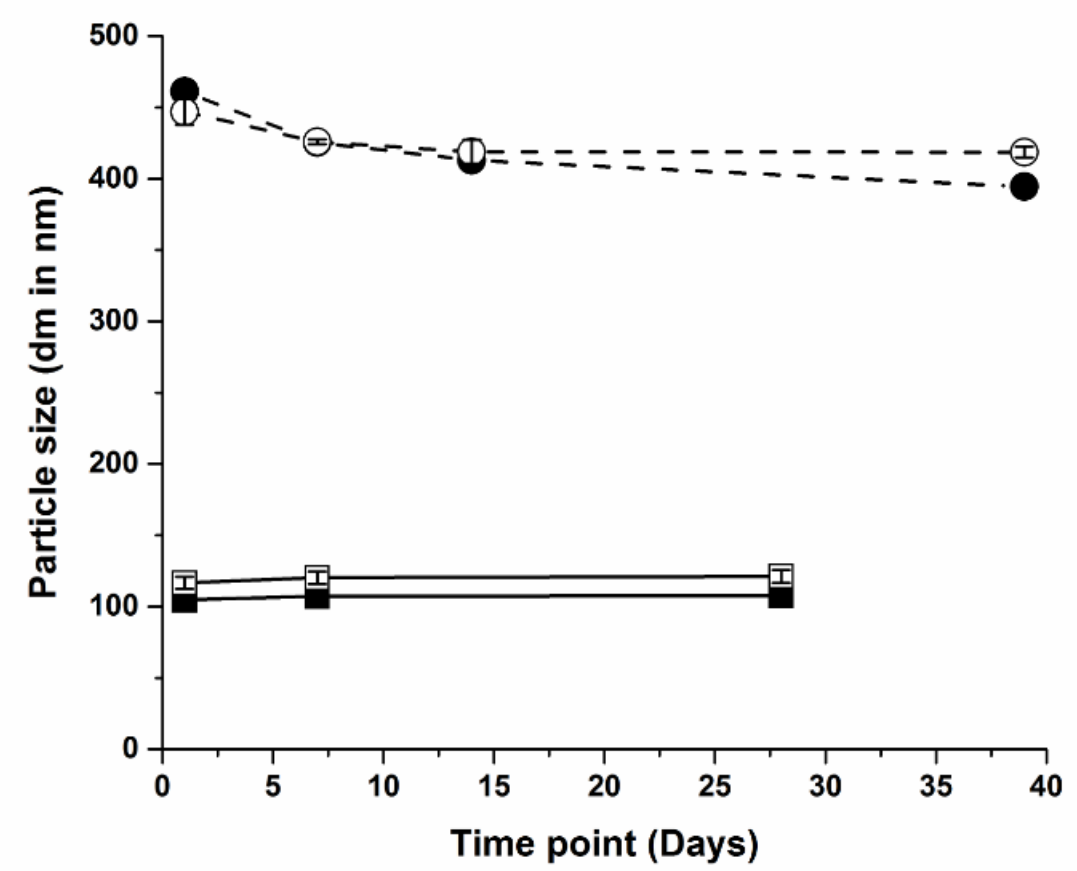

a)

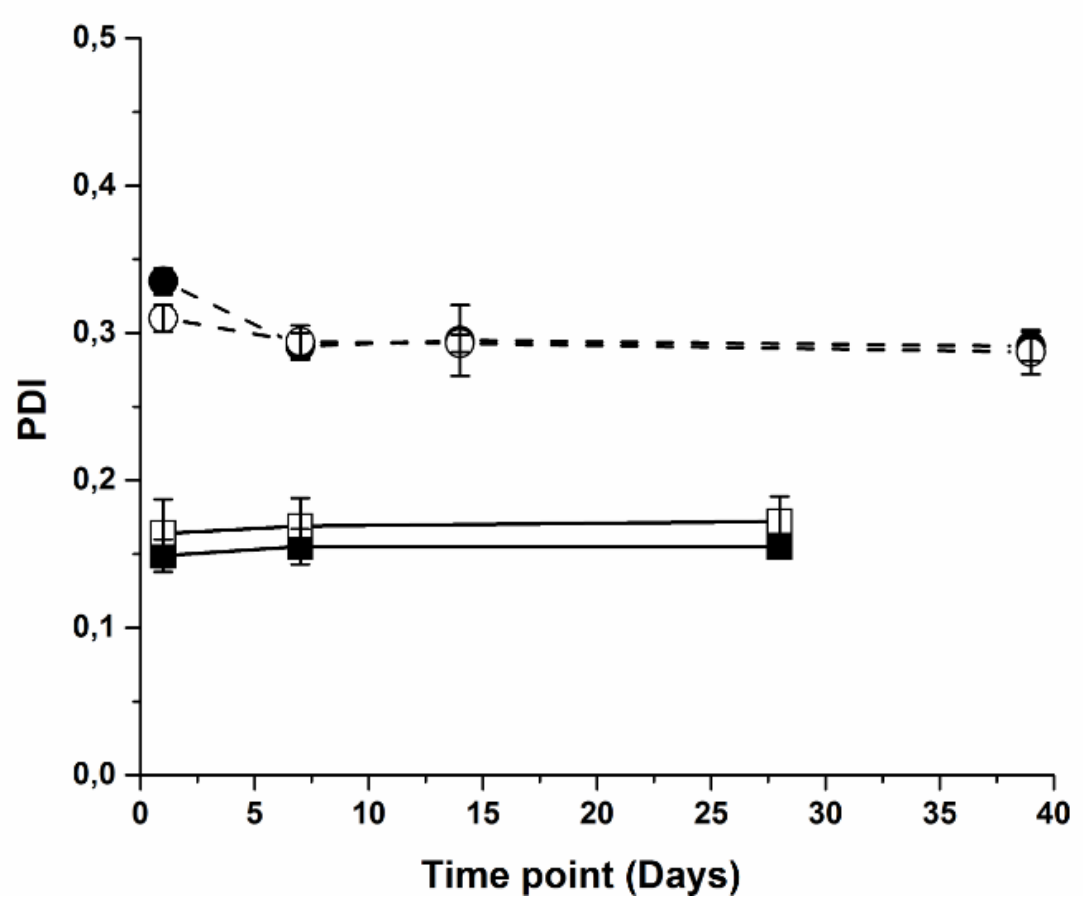

Figure 2: Changes in the a) particle size and b) PDI of fluoride loaded polymeric nanoparticles with time when stored in the refrigerator: Chitosan nanoparticles (solid lines) and pectin nanoparticles (dashed lines) prepared in $0.2 \% \mathrm{NaF}$ (open symbols) and $0.4 \% \mathrm{NaF}$ (solid symbols), respectively. Error bars denote standard deviations, $\mathrm{n}=6$. 


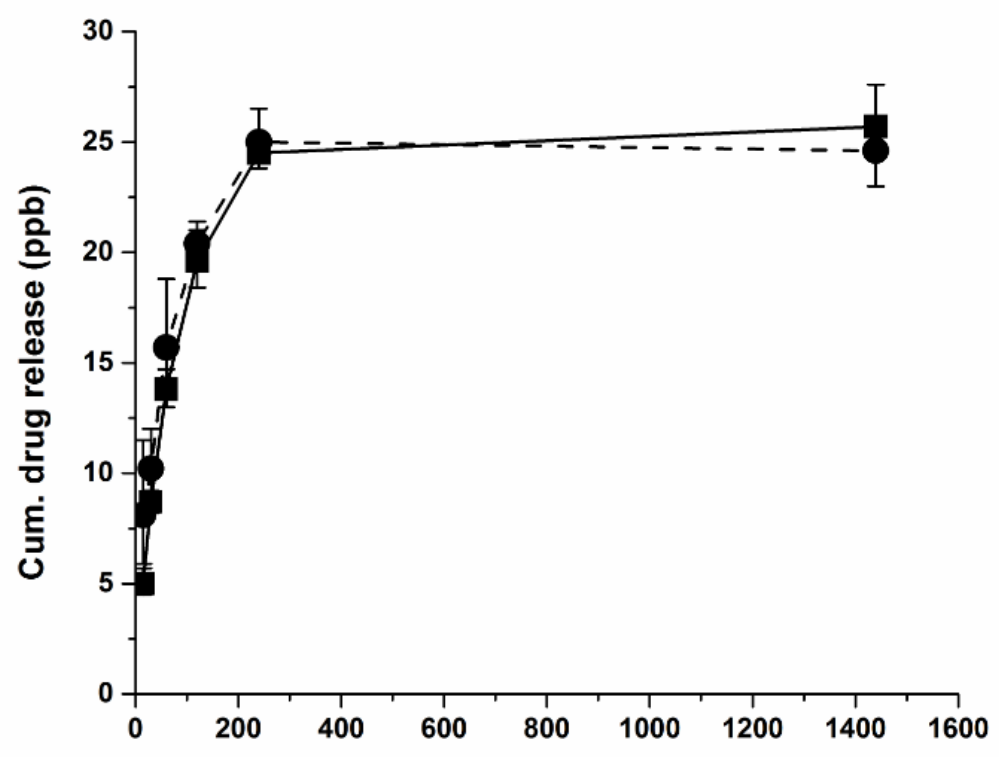

a)

Timepoint (Min.)

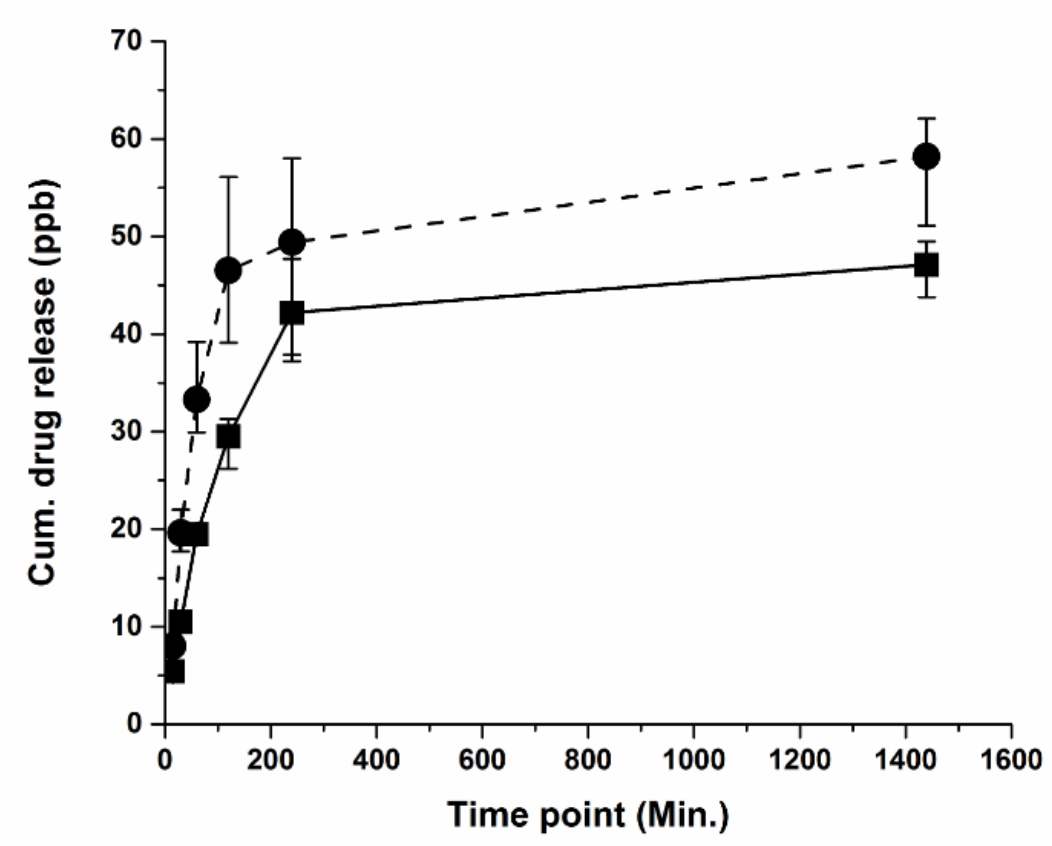

b)

Figure 3: Cumulative fluoride release from chitosan nanoparticles prepared in a) $0.2 \% \mathrm{NaF}$ and b) $0.4 \% \mathrm{NaF}$ in $10 \mathrm{mM}$ phosphate buffer $\mathrm{pH} 7$ (solid lines) and $\mathrm{pH} 5$ (dashed lines), respectively. Error bars denote maximum and minimum values, $\mathrm{n}=3$. 